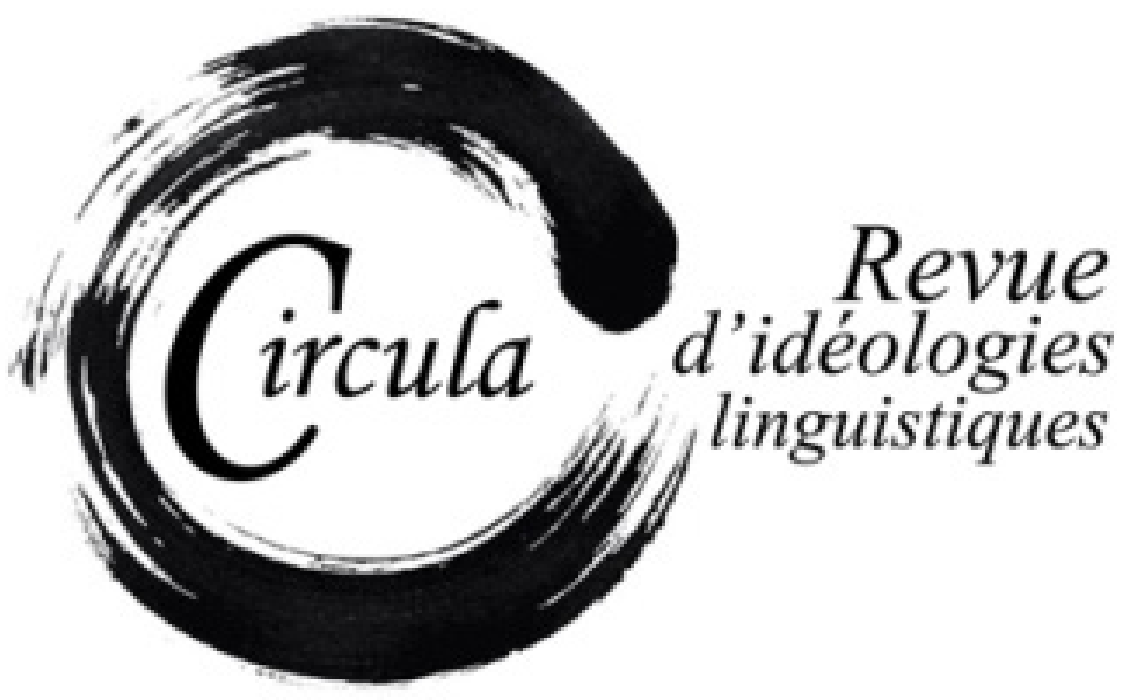

TITRE: LE RÔLE DE LA PRESSE DANS LA DISCUSSION DE LA RÉFORME DE L'ORTHOGRAPHE FRANÇAISE Auteur(s): AnNA EWIG, UnIVERSitÄT MÜnSter

Revue: Circula, NumÉro 7

PAGES: $70-89$

ISSN: 2369-6761

DIRECTEUR: WIM REMYSEN

URI: HTTP://HDL.HANDLE.NET/11143/14495

DOI: HTTPS://DOI.ORG/10.17118/11143/14495 


\section{Le rôle de la presse dans la discussion de la réforme de l'orthographe française}

Anna Ewig, Universität Münster anna.ewig@uni-muenster.de

Résume : Cet article a l'objectif d'étudier les réactions de deux quotidiens français - Le Monde et Le Figaro - à la dernière réforme de l'orthographe française proposée en 1990. Les questions qui se posent sont multiples: Quelle a été la visibilité de cette réforme au sein de cette presse écrite? Quelles en ont été les modalités linguistiques et discursives? Quels ont été les acteurs du discours porté sur les rectifications? Cette presse a-t-elle porté le poids de la question délicate de l'idée d'une réforme de l'orthographe en général ou s'est-elle concentrée plus précisément sur les changements proposés en 1990 ? Sur le plan méthodologique, une approche chronologique a été choisie afin de respecter la structure temporelle du débat et de pouvoir en appréhender l'évolution. II s'agit de trois périodes représentatives pendant lesquelles une discussion sur cette réforme eut lieu en France, à savoir 1990, 2009 et 2016.

Mots-clés : réforme de l'orthographe ; rectifications de l'orthographe française de 1990 ; articles de presse; idéologies linguistiques

Abstract: This article aims to study the reactions of two French newspapers - Le Monde and Le Figaro - to the most recent reform of the French orthography which was published in 1990 . The questions treated concern the visibility of this reform in the written press, its linguistic and discursive modalities, the principal actors of the discourse on the reform and the capacity of the newspapers to concentrate on a concrete reform fading out the idea of a reform in general. Methodologically, a chronological approach has been chosen in order to respect the temporal structure of the debate and to understand its evolution. Three representative periods have thus been selected during which a discussion of this reform has taken place in France, i.e. 1990, 2009 and 2016.

Keywords: orthography reform; rectifications de l'orthographe française of 1990; newspaper articles; linguistic ideologies 


\section{Introduction}

L'orthographe française est un système complexe qui délivre - à qui sait les comprendre - des renseignements divers sur les mots : prononciation actuelle et d'antan, informations grammaticales, étymologie, parenté avec d'autres mots ou langues, différenciation d'homonymes, erreurs d'interprétation historiques, etc. Cette spécificité qui rend son apprentissage long et éprouvant a abouti à l'émergence d'une admiration, voire d'une idolâtrie de l'orthographe1, d'où la difficulté de la réformer. Des réformes ont néanmoins été proposées régulièrement au cours de son histoire, la dernière étant les rectifications de l'orthographe du 6 décembre 1990. Celles-ci ont été élaborées par un groupe de travail et un comité d'experts constitués par le Conseil supérieur de la langue française créé en 1989 dans lesquels collaboraient entre autres l'Académie française, des écrivains, les maisons d'édition Robert et Larousse ainsi que plusieurs linguistes. Les rectifications portent sur les points suivants:

- le trait d'union et la soudure de quelques mots

- le pluriel des mots composés et des emprunts

- les accents (dont le circonflexe) et le tréma

- la simplification des consonnes doubles

- l'accord du participe passé de laisser suivi d'un infinitif

- quelques anomalies (dont nénufar, ognon)

- des recommandations aux lexicographes

La connaissance même de cette réforme de 1990 demeure jusqu'à aujourd'hui partielle au sein de la société française, non seulement pour la moyenne des francophones mais aussi pour les élèves et enseignants, soit les deux groupes de la population les plus directement concernés par l'apprentissage de l'orthographe. Pour tenter de comprendre le destin de cette réforme, la presse, cet espace médiatique dans lequel les diverses opinions d'une société s'expriment de manière interposée, nous apparaît être un espace d'analyse incontournable. Puisque dans le passé, on a pu constater une participation active de la presse dans l'échec de réformes orthographiques par son influence sur l'opinion publique et politique ${ }^{2}$, cet article propose de traiter de son rôle dans le destin des dernières rectifications. La presse écrite prend part à la discussion de cette réforme de deux façons : d'un côté,

1. En France, notons que l'exercice scolaire de la dictée coexiste sous forme de compétitions (amicales) collectives. Cet exercice consistant à vérifier la maîtrise de l'orthographe est donc devenu une passion collective volontaire à caractère quelque peu sportif. Les fameuses dictées de Pivot en sont un exemple. Feschet (1998: 191) dit à cet égard que « [l]'orthographe est souvent perçue comme un système de valeurs dont la pratique s'apparente plus à un acte de foi qu'à un usage fonctionnel. »

2. P. ex. au moment des arrêtés de tolérances de 1900/1901, qui n'étaient certes pas des réformes orthographiques proprement dites mais qui auraient pu - et c'était une des idées de leurs promoteurs - influencer l'usage à long terme et ainsi aboutir à une évolution de l’orthographe (Keller, 1991 : 7s.). 
en prenant le double rôle de l'informatrice-commentatrice, de l'autre, en favorisant dans ses publications une des orthographes en vigueur. Alors que le dernier point ne nécessite pas de commentaire approfondi, les quotidiens considérés ici optant pour l'orthographe traditionnelle, le premier point sera étudié en regardant de près des articles et commentaires des deux quotidiens français les plus importants à l'échelle nationale et représentant deux orientations politiques différentes, Le Monde et Le Figaro. Nous nous concentrerons sur la France pour deux raisons : d'une part, l'initiative de mettre en place le Conseil supérieur de la langue française pour élaborer ces rectifications avait été prise par Michel Rocard, alors premier ministre français. D’autre part, contrairement aux autres pays francophones d'Europe, la France - et par là nous entendons les responsables de l'éducation - a pendant longtemps ignoré cette réforme ${ }^{3}$. Ce n'est en effet qu'en 2008 - presque 18 ans après leur publication - que les rectifications sont mentionnées dans les programmes scolaires en France, et ceci avec un certain manque de clarté et de pragmatisme. Pour l'école primaire, on peut y lire que «l'orthographe révisée est la référence » (Ministère de l'Éducation nationale et Ministère de l'Enseignement supérieur et de la Recherche, 2008 : 37), une formulation très imprécise. On n’y trouve ni définition du terme orthographe révisée ni explication du cadre de sa fonction de référence. Cette phrase ne nous apprend donc rien sur l'application concrète de la réforme : la question de savoir si la nouvelle orthographe doit être enseignée activement ou juste tolérée pendant les examens officiels demeure floue. Pour le collège, les programmes scolaires sont un peu plus clairs, du moins en ce qui concerne la dénomination de la réforme. On peut y lire que « [p]our l'enseignement de la langue française, le professeur tient compte des rectifications de l'orthographe proposées par le Rapport du Conseil supérieur de la langue française, approuvées par l'Académie française » (Ministère de l'Éducation nationale, $2008: 2)^{4}$. Si la réforme est clairement indiquée, il n'est pas précisé dans quelle mesure les professeurs sont amenés à « tenir compte » de celle-ci : La réforme devrait-elle être enseignée de manière explicite et proactive, mentionnée en cours de manière non-systématique, tolérée

3. En Belgique, le ministère de l'Éducation informe les enseignants dès 1990 par une lettre officielle de l'existence des rectifications en précisant que « les deux orthographes auront à coexister et seront acceptées » (CSLF, 2005 : 8). En 1998, une circulaire ministérielle reconfirme cette position, une autre, de 2008, recommande d'enseigner prioritairement les nouvelles formes (Legros et Moreau, 2012 : 32). En Suisse, la Conférence intercantonale de l'instruction publique (CIIP) publie en 1996 une brochure d'information sur les rectifications accompagnée d'une circulaire demandant aux enseignants de ne pas tenir pour fautive ni la nouvelle ni l'ancienne orthographe (CIIP, 1996). Cette brochure est actualisée en 2002. Pour ne pas passer sous silence la situation au Québec, le ministère de l'Éducation y attend l'année 2009 pour diffuser une note indiquant que « les élèves qui utilisent les graphies traditionnelles ou les nouvelles graphies ne seront pas pénalisés dans le contexte des corrections effectuées par le Ministère » (MELS, 2009). À ce jour, il n'y a pas d'autres consignes officielles concernant l'application de la réforme au Québec. Les effets de ces directives officielles se reflètent dans les résultats de deux études empiriques : le taux de connaissance et d'application des rectifications est d'après un sondage de Biedermann-Pasques (2006) plus élevé chez les étudiants belges et suisses que chez leurs homologues français et québécois. Ce sondage ayant été effectué en 2002 et 2003, la majorité des personnes consultées a d'après leur âge été scolarisée peu après la publication des rectifications et aurait donc pu être en contact avec la nouvelle orthographe dès le début de leur apprentissage de l'orthographe - si elle leur était enseignée. Un autre sondage effectué en 2010 montre une réalité comparable malgré quelques évolutions, notamment une nette augmentation des résultats en faveur des rectifications (Groupe RO, 2012). La France occupe la dernière place dans les deux sondages en termes de connaissance et d'application de la réforme.

4. À partir de 2015, une formulation similaire se trouve dans le programme de l'école primaire (Ministère de l'Éducation nationale, 2015). 
dans les examens, ... ? Cela laisse à supposer que c'est à chaque professeur de décider du sort des nouvelles formes de l'orthographe dans ses cours. À en croire un sondage de 2002 et 2003 effectué auprès de 306 étudiants dans quatre pays francophones, ce sort est plutôt négatif : seulement 10\% des étudiants français disent connaître les rectifications (Biedermann-Pasques, 2006 : 13). Si - dans un test intégré dans ce sondage - 30\% des nouvelles formes sont utilisées par ces mêmes étudiants, ce n'est probablement pas parce que celles-ci leur avaient été enseignées pendant leur scolarité mais par analogie avec des règles préexistantes. En effet, la plupart des nouvelles formes utilisées dans ce test concerne la régularisation du pluriel des mots composés (application du morphème du pluriel en finale du deuxième élément du composé, comme un cure-ongle, des cure-ongles), tandis que des formes anomales mais à un degré de reconnaissance élevé comme oignon (au lieu d'une forme rectifiée ognon sans $\langle i$ ) sont conservées. Dans un autre sondage plus récent effectué auprès de 361 étudiants et enseignants dans six pays francophones, on peut constater une augmentation de la connaissance des rectifications pour la France (Groupe RO, 2012). Malgré tout, la réforme demeure peu connue puisque seulement un peu moins du quart des personnes interrogées affirment la connaître et qu'une quantité avoisinante de personnes indique n'en avoir jamais entendu parler. Plus de la moitié ne la connaît que très vaguement. Quant aux seuls enseignants, le taux de connaissance s'élève à 38,5\%. Une autre question du sondage concerne l'enseignement de quelques-unes de ces nouvelles règles par les enseignants interrogés. Environ 59\% des formes enseignées par les enseignants français se limitent à l'ancienne orthographe. On peut donc constater avec les auteurs du sondage que « la connaissance des rectifications va de pair avec leur enseignement» (Groupe RO, 2012: 143).

\section{La presse et sa discussion de la réforme}

Pour en revenir à notre problématique qui est l'étude du traitement de cette réforme dans la presse, nous avons opté pour deux journaux - Le Monde et Le Figaro - qui comptent parmi les quotidiens français les plus lus et qui représentent deux orientations politiques différentes : alors que Le Figaro se positionne à droite / centre-droit et vise un lectorat libéral-conservateur, Le Monde, malgré sa ligne éditoriale neutre, possède un lectorat plus positionné à gauche. De plus, notons que Le Figaro avec sa rubrique "Le bon français », qui existe depuis 1996, montre une préoccupation importante - et conservatrice - pour des questions linguistiques 5 . On peut donc s'attendre à deux positionnements différents sur la réforme.

Dans notre étude, une approche chronologique a été privilégiée afin d'appréhender l'évolution du débat. Nous nous concentrerons ainsi sur trois étapes clés: le mois de décembre 1990, le mois suivant la publication des rectifications ; septembre et octobre 2009, les six semaines qui rassemblent les réactions à la parution d’un livre du journaliste François de Closets se prononçant, dans un

5. Dans son étude portant sur les réactions aux rectifications faites par les lecteurs dans les forums de discussion de différents journaux, Vicari (2016: 112s.) constate cette même préoccupation chez les lecteurs du Figaro qui sont beaucoup plus nombreux à réagir à des articles que ceux du Monde. 
langage accessible à tous, pour une meilleure mise en pratique de la réforme ; et finalement, février 2016 qui correspond à l'annonce faite par des éditeurs de manuels scolaires d'appliquer la réforme à la rentrée 2016/2017. Dans ces trois périodes, un nombre total de 90 articles traitant d'une réforme de l'orthographe a pu être trouvé dans ces deux journaux. Quant à l'analyse du contenu des articles, notre approche, principalement qualitative - elle portera sur la qualité de la présentation des rectifications, le lexique utilisé, les argumentations déployées et la qualité et identité des intervenants -, se veut également quantitative puisque l'intégralité des articles sera recensée et catégorisée. Nous verrons comment et dans quelle mesure la réforme a été thématisée dans ces journaux. Les questions qui se posent sont les suivantes: Dans quelle mesure la presse réalise-t-elle son rôle d'informatrice? De quelle manière commente-t-elle les évènements présentés et à quels acteurs donne-t-elle la voix ? Se positionne-t-elle du côté d'un des partis ou reste-t-elle neutre? Et finalement, peut-on constater une évolution de ces points entre 1990 et 2016 ?

\subsection{Décembre 1990}

En décembre 1990, après la publication des rectifications, une discussion sur l'orthographe et sa réforme s'enflamme littéralement. Cette discussion mène à la fondation de deux organisations qui appellent à protester contre la réforme et qui comptent parmi leurs membres un nombre grandissant de personnalités de la vie publique ${ }^{6}$. Vers la fin de décembre, quelques membres de l'Académie française commencent à critiquer publiquement certains points de la réforme, qui avaient pourtant été approuvés unanimement par l'Académie elle-même en mai 1990. Le caractère exact de ce vote unanime - seuls 22 des 38 académiciens y étaient présents ${ }^{7}$ - devient par la suite le sujet d'un débat lancé par Bernard Pivot, lui-même membre du Conseil supérieur de la langue française et donc personnellement actif à l'élaboration de la réforme. Il est reproché au secrétaire perpétuel de l'Académie, Maurice Druon, d'avoir menti sur la question de l'unanimité et d'avoir ainsi contribué à l'officialisation de la réforme grâce à la bonne réputation de cette institution.

Tous ces évènements sont repris dans les articles de presse de décembre 1990. Cela est principalement le cas pour Le Figaro, qui publie 32 articles et commentaires sur le sujet, dont sept qui délivrent des présentations objectives de la réforme et les 25 restants qui font preuve d'une attitude négative de la part des auteurs ou des personnes qui y sont mentionnées. Le Monde se montre plus modéré avec seulement six articles, dont quatre qui abordent la réforme d'une manière plus objective en présentant son contenu ou les discussions évoquées par elle et deux articles qui donnent la voix à un défenseur et à un opposant de la réforme.

6. À titre d'exemple on peut citer des adhérents journalistes, écrivains, philosophes comme François Cavanna, Alain Finkielkraut, Françoise Sagan ou Michel Tournier, mais aussi des lauréats de Prix Nobel de matières plus éloignées des lettres comme le physicien Louis Néel ou le biologiste André Lwoff, ou encore le fondateur de l'une de ces associations, le futur ministre de l'Éducation François Bayrou.

7. Ce chiffre est tout à fait représentatif pour la présence habituelle des académiciens. 
En ce qui concerne plus en détail leur contenu, on peut remarquer qu'après une courte phase initiale pendant laquelle les informations se centrent sur les points orthographiques concernés par les rectifications - sans pour autant jamais donner une présentation complète des quelques règles ${ }^{8}$-, ce qui domine est la présentation de la discussion qui a lieu entre les défenseurs et les opposants, tout en privilégiant le point de vue des détracteurs.

Quant à la réception de la réforme, c'est dans ce contexte d'opposition que le champ lexical de la guerre et du combat est utilisé afin de dénoncer la violence prétendue de la réforme ou de se distancier de l'émotion suscitée, pas toujours sans humour comme dans les deux exemples suivants:

Et ceux qui verraient un petit parfum d'adultère à cette liaison de la politique et de la littérature se trompent: le but de l'alliance n'est autre qu'une croisade contre « la procédure autoritaire qui tend à imposer, en prenant les apparences extérieures du droit, une nouvelle orthographe (...) arbitraire et sans justification objective », dont le seul effet sera de déconsidérer «la langue écrite et ses règles ». (Raphaëlle Rérolle, le 20 décembre 1990, Le Figaro)

On se chamaille entre immortels à coups de traits d'union, tandis que les professionnels de la langue affûtent leurs accents circonflexes. (Philippe Bernard, le 31 décembre 1990, Le Monde)

En ce qui concerne Le Figaro, une grande partie des articles emploie ce type de vocabulaire de manière répétitive marquant par là un rapport plus étroit, plus émotionnel voire personnel à cette réforme. Cette récurrence précise son aspect historique prétendu, la justification du combat n'en devenant que plus logique. Pour certains, cette réforme met un terme à un long conflit et pour d'autres, elle le ravive ou l'exacerbe:

Aujourd'hui, le premier ministre, d'un trait de plume, met un point final à la guerre de cent ans de l'orthographe. (Georges Suffert, le 6 décembre 1990, Le Figaro)

Orthographe : à fleurets mouchetés. Partisans et adversaires de la réforme s'affrontent. Le duel qui oppose le Conseil supérieur de la langue française à la Société des agrégés se poursuit pour « Le Figaro ». (Jacques Malherbe, Muriel Frat et Véziane de Vezins, le 10 décembre 1990, Le Figaro)

La trêve des confiseurs n'aura pas réussi à calmer la bataille déclenchée par les modifications de l'orthographe. (Muriel Frat, le 26 décembre 1990, Le Figaro)

C'est dans ce contexte français qu'apparaît à plusieurs reprises le motif historique de la résistance face à l'occupation nazie en France, une des associations anti-rectifications s'intitulant « Le français

\footnotetext{
8. Le caractère incomplet des informations données aux lecteurs peut être envisagé comme une des raisons de l'éventuel échec de la réforme : plus le public est informé, plus il est favorable aux rectifications, du moins en théorie (Groupe RO, 2012 : 132). On trouve d'ailleurs dans notre corpus la citation du secrétaire général du Syndicat national des instituteurs et des professeurs de collège de l'époque, qui remarque que « dans le détail, les propositions ont été mal expliquées. La présentation qui a été faite donne le sentiment que la réforme est profonde alors que ça n'est pas le cas. Ce qui manque, c'est une explication. » (citation de Jean-Claude Barbarant par Muriel Frat, le 31 décembre 1990, Le Figaro)
} 
libre ». Les rôles du bon et du méchant sont ainsi clairement répartis et incompatibles. Il est demandé au lectorat de choisir son camp :

Une vingtaine d'auteurs prestigieux [...] ont accepté de soutenir François Bayrou dans sa croisade contre la réforme [...]. Des journalistes et des éditeurs ont également décidé de se joindre aux écrivains. Leur point commun : l'esprit de la résistance. [...] Les prestigieux adhérents de l'association - Le français libre - lancent un manifeste de résistance dans lequel ils expliquent qu'ils n'appliqueront la réforme pour rien au monde. [...] Nombreux sont d'ailleurs les maitres qui partent en guerre contre les modifications du Conseil supérieur de la langue française. (Muriel Frat, le 18 décembre 1990, Le Figaro)

Orthographe : la résistance s’organise (Muriel Frat, le 19 décembre 1990, Le Figaro)

La bataille engagée par les défenseurs de la langue française n'est pas gagnée d'avance. Elle se jouera, en fait, dans les classes où les instituteurs décideront d'enseigner ou non les « rectifications ». Et dans les familles qui choisiront la soumission ou la résistance. (Muriel Frat, le 19 décembre 1990, Le Figaro)

Finalement, le combat est transposé à une civilisation passée. C'est l'image des gladiateurs de la Rome antique osant se battre pour la gloire (de la langue française) qui est évoquée dans le titre de l'article suivant:

« Deux écrivains dans l'arène ». (Véziane de Vezins, le 14 décembre 1990, Le Figaro)

Les articles dessinent ainsi l'image d'une profonde désunion, en partie apocalyptique, par laquelle la France, la francophonie et la langue française sont menacées à cause des rectifications. Le rayonnement de la France semble en danger:

Dans les pays francophones comme en France, les « rectifications » vont entraîner une belle pagaille. Elles risquent même, selon certains spécialistes, de nuire à la diffusion du français et de détourner d'une langue dont on ne connaîtra plus les règles précises. (Muriel Frat, le 24 décembre 1990, Le Figaro)

On s'inquiète aussi des conséquences qu'auront les rectifications pour la population, notamment pour les élèves et les autres scripteurs ordinaires. Les rectifications seraient source de confusion et d'augmentation des inégalités sociales, toutes les deux causées par la coexistence des deux orthographes et par l'introduction d'exceptions dans les nouvelles règles:

Devenue facultative, la réforme de l'orthographe semble perdre beaucoup de sa nocivité : mais ne risque-t-elle pas de semer inutilement la confusion dans l'esprit des élèves? (Muriel Frat et Jacques Malherbe, le 6 décembre 1990, Le Figaro) 
François Bayrou : [...] les principales victimes seront, une nouvelle fois, les plus faibles, les gens qui ont fait un effort pour connaitre la langue et qui vont se mettre à douter de tout. La langue doit être un lieu de rassemblement, pas un lieu de discrimination entre ceux qui suivent des règles et ceux qui en suivent d'autres. (Muriel Frat, le 11 décembre 1990, Le Figaro) Croit-on que les décideurs, issus eux aussi, et pour longtemps encore, des écoles de l'ancienne orthographe, donneront pas, à égalité de valeur, la préférence aux candidats qui se diront combatifs, à l'ancienne, plutôt que combattifs, et à ceux qui aiment mieux les nénuphars que les nénufars ? [...] Ainsi aura-t-on créé une barrière de plus dans ce monde qui se voudra toujours plus ouvert, plus accueillant, plus attaché à l'égalité de tous. Pis qu'une barrière, un mur, une muraille. Un racisme. Le racisme anti-nénufar. (François Crouzet, le 19 décembre 1990, Le Figaro)

Les points de réforme évoqués dans les articles et les commentaires se ressemblent, et ce sont surtout l'accent circonflexe - devenu facultatif sur les i et les u dans la plupart des cas - et à un moindre degré la soudure de certains mots composés ainsi que les graphies nénufar et ognon qui deviennent les emblèmes négatifs des rectifications:

Avec la permission du secrétaire perpétuel de la Compagnie à laquelle j'ai l'honneur d'appartenir, je verserai un pleur furtif sur quelques mots de notre langue, décoiffés de leur accent circonflexe par la nouvelle orthographe. (André Frossard, le 7 décembre 1990, Le Figaro)

Plusieurs immortels ont emboîté le pas à Jean d'Ormesson, à Alain Peyrefitte et à Maurice Rheims pour dénoncer la suppression de l'accent circonflexe et les nouvelles règles des mots composés. (s.a., le 21 décembre 1990, Le Figaro)

Bien sûr, les millions de contestataires n'occuperont pas le pavé de Paris et des principales villes de France pour réclamer à Michel Rocard la restauration de l'accent circonflexe ou du trait d'union. (Muriel Frat, le 26 décembre 1990, Le Figaro)

Une grande partie des articles du Figaro donne la voix aux célèbres adversaires de la réforme. Les exemples et arguments de ces derniers qui sont évoqués dans les articles pour plaider contre les nouvelles formes ne sont pas toujours corrects, un point qui n'est jamais rectifié par les journalistes. Ainsi, à côté d'une mission d'information du public à moitié accomplie par la presse - comme mentionné plus haut, la réforme n'est jamais présentée de manière complète malgré sa faible envergure -, on trouve une certaine désinformation des lecteurs. La disparition complète de l'accent circonflexe - information incorrecte - cristallise les peurs et les critiques :

Et l'âme, la pauvre âme! Surmontée de son toit de pagode, ou de son hirondelle typographique, elle montait d'elle-même vers le ciel. Maintenant, elle ne pourra plus que raser le ciel, il lui manquera l'inspiration, autrement dit le souffle. (André Frossard, le 7 décembre 1990, Le Figaro) 
«Mais je suis farouchement hostile à la disparition de l'accent circonflexe. [...] On ne prononce pas de la même manière « le bât blessé » et les «bas » que portent les femmes. Pour une fois que la graphie n'est pas entièrement gratuite, qu'elle correspond à quelque chose, je trouve stupide de prendre une telle disposition. » (citation de Robert Merle par Muriel Frat, le 28 décembre 1990, Le Figaro)

Finalement, c'est encore dans les énonciations des adversaires qu'on remarque fréquemment une confusion entre l'orthographe et la langue - ne prenant pas en considération le fait que l'orthographe est un système construit dont l'évolution n'est pas naturelle mais bien le résultat de décisions prises par une partie de la communauté linguistique, ou jugeant qu'un changement apporté à l'orthographe est nuisible à la langue parlée, celle des générations précédentes et des voisins francophones:

«Deux choses me hérissent dans cette affaire, déclare Gérard Blua, organisateur du collectif et directeur de la petite maison d'édition marseillaise, le fait qu'un ministère décide, du jour au lendemain, que les enfants ne parleront plus la langue de leur parents. [...] » (s.a., le 19 décembre 1990, Le Figaro)

C'est une publication du Journal officiel qui va expliquer aux Belges francophones comment ils doivent parler. C'est étrange et même choquant. (citation d'Hervé Hasquin par Muriel Frat, le 24 décembre 1990, Le Figaro)

Peu concerné par les rectifications, Louis Leprince-Ringuet estime, pour sa part, qu' «il faut laisser la langue se créer, se faire toute seule et les mots étrangers se franciser avec le temps. » (Muriel Frat, le 31 décembre 1990, Le Figaro)

Un point régulier de désaccord et de critique est le fait que la réforme est décidée et imposée par le gouvernement, déviant ainsi du cours jugé naturel de la langue française. Selon certains détracteurs de la réforme, l'orthographe serait déterminée par l'usage et non par les instances de sa régulation et de sa fixation:

François Bayrou : [...] Nul n’a le droit de toucher à la langue française parce qu'elle n’appartient à personne. (Muriel Frat, le 11 décembre 1990, Le Figaro)

Michel Tournier : L’orthographe dépend de l'usage. Personne en France n'est habilité à commander l'usage, ni le président de la République, ni l'Académie française. [...] je trouve extraordinaire de vouloir changer l'orthographe par décret. (Véziane de Vezins, le 14 décembre 1990, Le Figaro)

La langue est un phénomène collectif et démocratique par excellence. Il est l'expression même de la fameuse société civile. On ne la transforme pas par décret. Les décrets ne peuvent rien faire d'autre que de reconnaître un usage préexistant. Qui réforme une langue, une grammaire, une orthographe? Ni le gouvernement, ni les syndicats, ni un comité, ni même un haut comité, ni même l'Académie. C'est le peuple. (Jean d'Ormesson, le 22/23 décembre 1990, Le Figaro) 
Nous l'avons constaté, la manière dont la réforme est présentée dans la presse après sa publication joue un rôle décisif dans le sort de celle-ci. À la suite des débats médiatiques échauffés, l'Académie française, qui avait pourtant approuvé à l'unanimité la réforme en mai 1990 après une présentation des points orthographiques concernés, commence à remettre en question certains points de son contenu à partir de la fin du mois de décembre. En janvier 1991 un nouveau vote a lieu, la réforme est de nouveau approuvée (Catach, 1991 : 18-20), mais les académiciens demandent en même temps qu'elle « ne soi[t] pas [mise] en application par voie impérative et notamment par circulaire ministérielle ». Plutôt, ils souhaitent que « ces simplifications et unifications soient soumises à l'épreuve du temps » (Académie française, s.d.), sans préciser la durée de temps nécessaire pour savoir si telle ou telle forme est entrée dans l'usage ou pas. Sans préciser non plus comment une forme orthographique qui n'est pas enseignée et qui ne figure pas dans les dictionnaires - car seules les formes déjà en usage y figurent -, est censée entrer dans l'usage. À la suite de ces évènements seront oubliés les plans du comité d'experts qui consistaient à faire enseigner les rectifications aux nouveaux élèves à partir de la rentrée 1991/92 (Goosse, 1991 : 30s.).

\subsection{Septembre / octobre 2009}

L'épreuve du temps fit quelque peu tomber les rectifications dans l'oubli de l'opinion publique. Peu de temps après leur publication, on présume déjà leur échec (Arrivé, 1993 : 9 ; Walter, 1994 : 266). Toutefois, des étapes déterminantes - par exemple par l'intégration soudaine mais complète dans le Dictionnaire Hachette en 2002 et dans le Nouveau Littré en 2005², par l'intégration dans les programmes scolaires en 2008 mentionnée plus haut ou dans le correcteur informatique de Word en 2005 (Muller, 2006 : 253) - vont lui permettre de gagner en importance et en signification. De plus, les défenseurs de la réforme continuent leur travail d'information du public (p. ex. Masson, 1991 ; Contant et Muller, 2005 ; RENOUVO, 2005 ; Contant, 2009) et la recherche sur l'application des nouvelles règles (p. ex. CSLF, 2005 ; Honvault-Ducrocq, 2006 ; RENOUVO, 2006). Plusieurs associations s'occupent en France comme dans d'autres pays francophones de propager les rectifications : en France l'AIROÉ (Association pour l'information et la recherche sur les orthographes et les systèmes d'écriture, fondée par Nina Catach bien avant les rectifications en 1983), en Belgique l'APARO (Association pour l'application des recommandations orthographiques, fondée par André Goosse en 1991), en Suisse l'ANO (Association pour la nouvelle orthographe, fondée en 2001) et au Québec le GQMNF (Groupe québécois pour la modernisation de la norme du français, fondé en 2004). Ces associations sont aujourd'hui regroupées dans le RENOUVO (Réseau pour la nouvelle orthographe du français) et entretiennent entre autres des sites Internet pour fournir des informations au public ${ }^{10}$. Mais tous ces efforts se situent en dehors d'une discussion publique et médiatisée. Cette dernière

9. Le Petit Larousse illustré les suit en 2011. Le Petit Robert intègre aujourd'hui une partie mais non pas l'ensemble des nouvelles formes. Le dictionnaire de l'Académie française mentionne dans sa ge édition les formes rectifiées, mais cellesci ne sont souvent indiquées dans les articles que par un losange qui fait référence à une liste en fin de chaque volume.

10. ANO : www.orthographe-recommandee.info; APARO : sweet.ua.pt/fmart/aparo.htm ; GQMNF : www.gqmnf.org et www.nouvelleorthographe.info ; RENOUVO : www.renouvo.org. 
sera réactivée en 2009 par la publication du livre Zéro fautes. L’orthographe, une passion française de François de Closets retraçant l'histoire de l'orthographe française jusqu'à son évolution en « religion d'État » (de Closets, 2009 : quatrième de couverture) dans lequel il se prononce, entre autres, pour une meilleure mise en pratique de la réforme.

Si le changement des programmes scolaires en 2008 passe inaperçu dans Le Monde et Le Figaro, les deux journaux réagissent au livre de de Closets et à la discussion sur une simplification de l'orthographe à laquelle celui-ci a donné l'impulsion. Le Monde, il est vrai, dans une moindre mesure avec trois articles contre les huit articles, commentaires et présentations de sondages du Figaro dans les six semaines suivant la publication ${ }^{11}$. Tandis que les articles du Monde représentent de manière assez équitable les deux positions (pour/contre), Le Figaro se contente de deux articles sans parti-pris évident alors que les six articles restants thématisent principalement les côtés négatifs d'une réforme.

Il est intéressant de noter que la discussion porte avant tout sur le concept général d'une réforme de l'orthographe et non pas sur les rectifications de 1990, qui ne sont thématisées explicitement que dans trois articles. De plus, les points concrets nommés de cette réforme sont incomplets : en effet, ils concernent surtout la figure emblématique de l'accent circonflexe qui avait déjà dominé la discussion 19 ans auparavant:

A partir de la page 239, les chapitres [...] ne comportent plus [...] d'accents circonflexes : ils sont écrits en orthographe rectifiée. (Robert Solé, le 4 septembre 2009, Le Monde)

L'auteur raconte de manière hilarante les péripéties de la dernière réforme avortée de 1990, celle qui arrachait les accents circonflexes comme un vent mauvais fait s'envoler les chapeaux de cheminée. (Claude Duneton, le 15 octobre 2009, Le Figaro)

Cette dernière citation relève aussi d'une fausse information qui mène les lecteurs à penser que « le vent mauvais » des rectifications supprimerait tous les accents circonflexes. Deux autres informations de ce genre se retrouvent dans les articles. Le recours à la comparaison de la dernière citation a pour objectif d'alerter les lecteurs des risques et donc de compromettre tout esprit de réforme :

En revanche, la tentative de l'Académie française en 1990 - qui portait notamment sur la ponctuation et sur les accents circonflexes - a suscité un tollé qu'elle ne méritait pas. (Natacha Polony, le 3 septembre 2009, Le Figaro)

Et de citer l'Allemagne, qui a renoncé ces dernières années à sa réforme de l'orthographe : les Allemands ne comprenaient plus leurs journaux. (Natacha Polony, le 3 septembre 2009, Le Figaro)

Comme en 1990, on retrouve dans les articles le champs lexical du vocabulaire de guerre et du combat utilisé pour désigner la soi-disant montée d'intensité dans les quelques discours qui ont lieu:

11. Étant donné la plus faible résonance médiatique de ce livre - comparé à celle qui a eu lieu en 1990 et 2016 -, nous avons choisi un espace de temps plus large pour cette période-ci afin de posséder un plus grand éventail de réactions. 
En cette rentrée, l'essayiste François de Closets part en guerre contre une langue « figée » et des règles arbitraires. (Natacha Polony, le 3 septembre 2009, Le Figaro)

Dans son essai, Zéro faute, François de Closets évoque le combat entre « philographes » et " graphophobes » à côté de quoi la guerre entre Armagnacs et Bourguignons serait un pugilat de cour de récréation. (Étienne de Montety, le 17 septembre 2009, Le Figaro)

Et en rappelant la discussion de décembre 1990, un article résume:

On a assisté à une levée de boucliers. (Robert Solé, le 4 septembre 2009, Le Monde)

En somme, le livre de François de Closets provoque quelques réactions de la part de la presse dans lesquelles on retrouve certains éléments déjà évoqués en 1990 - sans pour autant mener à une discussion de fond sur les rectifications de l'orthographe qui n'étaient pourtant entrées dans les programmes scolaires que l'année précédant sa publication. Comme ces rectifications sont à peine thématisées, il n’est pas surprenant que leur contenu ne soit pas présenté de manière intégrale.

\subsection{Février 2016}

Plus près de nous dans le temps, le mois de février 2016 donnera une autre tournure à la discussion sur la question des rectifications, qui amènera même le secrétaire perpétuel de l'Académie française, Hélène Carrère d'Encausse, à se distancier de la réforme malgré son approbation par l'Académie en 1991 et sa prise en compte dans le dictionnaire académicien. Face à un article publié le 3 février sur le site de la première chaîne de télévision TF1 annonçant l'application de la réforme de 1990 à la rentrée 2016/2017, sans pour autant citer de sources pour cette information, la presse - et avant tout Le Figaro - s'empare du sujet. L'annonce de TF1 n'est pourtant pas entièrement correcte. II n'y avait aucune décision prise par les responsables de l'éducation nationale concernant l'enseignement de la < nouvelle > orthographe, contrairement à ce que l'annonce pouvait faire croire. II s'agissait en fait de quelques éditeurs de manuels scolaires qui après un quart de siècle avaient décidé d’appliquer les rectifications dans tous les manuels, certains les ayant déjà intégrées avant ${ }^{12}$. Le seul changement évident pour l'enseignement est par conséquent l'apparition systématique des nouvelles formes dans les manuels. Néanmoins, leur mise en application par les professeurs n'est nullement prescrite même s'il est probable que la nouvelle orthographe gagne en intérêt auprès des intéressés.

À regarder de plus près les réactions du Monde et du Figaro durant le mois de février, on constate que Le Monde se montre désormais un peu moins neutre qu'auparavant : parmi les douze articles et commentaires qu'il publie en février 2016, un tiers présente une image négative envers la réforme et seulement un sixième - soit deux articles - montre une attitude positive, les autres articles restant neutres. Quant au journal Le Figaro, celui-ci reste fidèle à sa ligne générale précédente avec 29 articles, commentaires et présentations de sondages, dont 17 le premier jour. Les deux tiers de

12. Voir la déclaration de la présidente des éditions Belin dans Le Monde (Samuel Laurent, le 4 février 2016, Le Monde). 
ces articles montrent une image négative de la réforme, un seul article la défend. Une fois de plus, l'information présentée dans les journaux ne porte pas tellement sur le contenu de la réforme, mais sur le débat qui l'entoure.

On retrouve ainsi à maintes reprises l'emploi du champ lexical de la guerre pour annoncer l'imminence du conflit provoqué par la réforme ou spécifier la particularité de son contexte culturel:

La bataille peut commencer! (Constance Jamet et Marie-Estelle Pech, le 4 février 2016, Le Figaro)

Circonflexes et traits d'union: la bataille de l'orthographe ravivée (Delphine de Mallevo, le 4 février 2016, Le Figaro)

Guerre de l’orthographe, angoisse existentielle typiquement française (s.a., le 16 février 2016, Le Monde)

En ce qui concerne la présentation du contenu des rectifications, les mêmes points concentrent l'attention et les critiques : il s'agit de l'accent circonflexe, des mots nénufar, ognon et des traits d'union. Ils serviront à identifier la réforme, à en signaliser la difficulté de la mise en application ou la superficialité de son intérêt :

«Ognon », «nénufar», accent circonflexe : l'orthographe se réforme. (s.a., le 4 février 2016, Le Figaro)

Chose amusante, les rédacteurs des programmes [scolaires] eux-mêmes ne sont pas parvenus à appliquer la réforme en intégralité, laissant passer de temps en temps un accent circonflexe sur « connaître » ou « maîtrise », ou bien parlant, en géographie, des « territoires ultra-marins » (en nouvelle orthographe, c'est ultramarin »). (Jean-Rémi Girard, le 4 février 2016, Le Figaro)

Car, sur le terrain, il n'est plus question d'accents circonflexes et de tirets, mais d'école délabrées, d'argent public gaspillé dans quelques portiques de sécurité imprudemment promis en campagne électorale, de disparition des bourses départementales aux collégiens ou de l'aide aux transports scolaires pour les familles... (Najat Vallaud-Belkacem, le 17 février 2016, Le Monde)

À nouveau, la confusion entre langue et orthographe fourmille. Pour les uns, les rectifications simplifient et donc dénaturent la langue française : une modification de l'orthographe entraîne une modification générale de la langue, c'est-à-dire sa dégradation. Pour les autres, elles corrigent les erreurs:

Les rectifications de l'orthographe de 1990 sont un ensemble de recommandations pour la simplification de la langue française. (Pierre Rubenach, le 4 février 2016, Le Monde)

[...] l'application des « Rectifications de l'orthographe du Conseil supérieur de la langue française, telles qu'elles sont apparues dans le Journal officiel du 6 décembre 1990, est un nouveau coup porté à la langue française. (Thierry Clermont, le 4 février 2016, Le Figaro) 
Devant les anomalies et manques de logique de notre écriture, en octobre 1989, le tout nouveau Conseil supérieur de la langue française, composé d'universitaires et de linguistes, est chargé par le premier ministre de réformer notre langue. (Marie-Estelle Pech, le 4 février 2016, Le Figaro)

De même, nous retrouvons des commentaires postulant que la rectification de la graphie de quelques mots va agrandir le fossé entre gens cultivés et ignorants. Le caractère égalitaire et unitaire présumé de la langue représente une source d'instabilité sociale:

Ainsi, on en arrive à faire coexister deux états de la langue, et l'on risque fortement de donner à chacun une valeur particulière car distinguer, c'est toujours à un moment ou un autre établir une classification entre supérieur et inférieur, dans ce genre d'affaires. Et tandis que certains professeurs consciencieux enlèveront les accents circonflexes, de méchants réactionnaires qui dirigent au plus haut niveau se feront un plaisir de faire de cette distinction un critère de jugement, voire un marqueur social inratable ou presque. (Jean-Rémi Girard, le 4 février 2016, Le Figaro)

Sous des dehors généreux, l'orthographe modernisée risque fort de pénaliser les plus faibles et de devenir un «marqueur social » discriminant, estime-t-il enfin. «Les familles aisée, les chefs d'entreprise conserveront leur attachement à l'orthographie actuelle. Ceux qui adopteront la nouvelle risquent fort d'être déphasés. » (citation d'Alain Bentolila par Marie-Estelle Pech, le 4 février 2016, Le Figaro)

A bien des égards, le monde risque de devenir bipartite : d'un côté, les riches de mots qui auront appris le latin ou le grec et orthographieront correctement; de l'autre côté, les pauvres de mots qui flotteront parmi les signes, en subissant le joug humiliant de ceux qui parleront et penseront à leur place. (Cécile Ladjali, le 18 février 2016, Le Monde)

Les opposants qui se prononcent contre les rectifications ou contre une réforme en général relient parfois l'orthographe à leur propre identité, aussi bien physique que mentale. L'apprentissage périlleux de l'orthographe est perçu comme une initiation constitutive de la construction personnelle:

Le but a beau être légitime, il n'empêche : c'est tout mon corps qui souffre. (Lucien Jedwab, le 10 février 2016, Le Monde)

Le français est une langue qui m'a malmenée longtemps et je crois que l'écriture m'est d'autant plus précieuse aujourd'hui qu'elle participe d'un combat. (Cécile Ladjali, le 18 février 2016, Le Monde)

L'histoire des mots, que la réforme ferait oublier, est également un sujet récurrent. Ce point avait déjà servi d'argument de manière sporadique dans les discussions en 1990 et en 2009, mais en 2016, il est plus régulièrement évoqué par les adversaires de la réforme:

L'accent de cime est tombé dans l'abîme. L'étymologie dit toute l'histoire d'une langue et d'un pays. Pas touche à mon circonflexe. (Marc de Boni, le 4 février 2016, Le Figaro) 
Faudrait-il, au nom d'une simplification dont l'urgence ne nous paraît pas évidente et les avantages contestables, sacrifier notre héritage ? [...] Les traces étymologiques adoucissent, le temps d'une découverte, l'indispensable, mais parfois inquiétant, arbitraire du signe. Elles révèlent la continuité et la cohérence de notre vocabulaire commun et nous rappellent qu'il est le résultat d’une évolution sans rupture. (Alain Bentolila, le 5 février 2016, Le Figaro)

Oublier l'histoire des mots, que l'orthographe révèle si bien, revient à renoncer à une partie de nous-mêmes. (Cécile Ladjali, le 18 février 2016, Le Monde)

Néanmoins, quelques articles, tous publiés dans Le Monde, dénoncent le côté polémique et irrationnel du débat:

Comment expliquer, alors, que le débat échappe à la rationalité ? 》 (Mattea Battaglia, le 16 février 2016, Le Monde)

La ministre de l'éducation nationale juge la polémique « absurde » et dénonce le rôle des anciens ministres de l'éducation nationale qui l'ont, selon elle, alimentée. » (Najat Vallaud-Belkacem, le 17 février 2016, Le Monde)

Au cours de ces derniers jours, pas moins de trois anciens ministres de l'éducation nationale et un ancien ministre de l'enseignement supérieur ont créé et alimenté une polémique absurde à propos d'une réforme de l'orthographe qui n'existe pas. » (Najat Vallaud-Belkacem, le 17 février 2016, Le Monde)

Nous noterons enfin cet article qui malgré un désaccord avec la réforme reconnaît que

« [c]'est [= les changements de l'orthographe] un peu triste, cela a un cout : le poétique. Mais rien d'essentiel n'est ici en jeu [...] ». (s.a., le 16 février 2016, Le Monde) ${ }^{13}$

Des présentations complètes de la réforme sont absentes des articles. Par contre, des erreurs persistent, notamment en ce qui a trait au circonflexe qui serait supprimé complètement d'après certains auteurs ou au mot nénufar qui perdrait avec le ph son origine étymologique présumée ${ }^{14}$ :

Cette réforme proposait une série de modifications : [...] suppression de certains particularismes, dont l'accent circonflexe. (Samuel Laurent, le 4 février 2016, Le Monde)

Parmi les principaux points, cette simplification des règles ne rend plus obligatoire l'accent circonflexe sur les « $u$ » et les « i ». (Constance Jamet et Marie-Estelle Pech, le 4 février 2016, Le Figaro)

13. L'auteur joue dans l'article avec l'emploi des nouvelles formes orthographiques, un jeu qui se reflète dans cette citation : regret un peu nostalgique des formes traditionnelles, moquerie d'une discussion sérieuse sur un sujet avec aussi peu d'envergure.

14. Cette graphie avec ph - due à une erreur d'étymologie - se trouve chez quelques lexicographes comme Nicot ou Richelet, mais jamais dans le dictionnaire de l'Académie française avant 1935 et peut ainsi être considérée comme une forme relativement récente dans l'orthographe actuelle. 


\section{Conclusion}

De manière générale, les lignes de conduite des publications du Monde et du Figaro se montrent assez différentes aussi bien au niveau quantitatif que qualitatif. Sur l'intégralité des trois périodes étudiées, Le Figaro a publié entre deux et cinq fois plus d'articles que Le Monde et a proposé un point de vue nettement plus tranché. En privilégiant les avis et arguments positionnés contre les rectifications, nous pouvons émettre l'hypothèse que le lectorat est plus directement visé, que ces articles le confortent dans sa position existante ou le convainquent $t^{15}$. Le Monde se montre quant à lui plus distancié et non partisan lors des deux premières périodes avant de publier, en 2016, un nombre d'articles plus conséquent et par la même occasion d'offrir pour la première fois une plus large place aux opposants de la réforme. Pourtant, c'est à ce moment même qu'il critique de manière ouverte la dimension irrationnelle du débat.

Malgré ces différences, les points communs entre les deux journaux existent. Parmi ceux-ci, quelques-uns sont observables à chaque moment de la discussion étudiée ici. Tout d'abord, on retrouve l'emploi du vocabulaire de guerre et du combat pour décrire le danger qui menacerait la langue française, mais aussi pour métaphoriser la confrontation entre les partisans et les adversaires de la réforme. Une telle intensité dans ce débat sur un sujet pouvant revêtir d'un point de vue extérieur relativement peu d'importance a soulevé l'étonnement de la presse internationale en 1990/1991, époque de conflits internationaux bien réels (la guerre du Golfe). Cela montre par là même l'envergure de l'émotion que suscite cette question en France. Ensuite, cette passion a embrasé le débat sur les rectifications notamment parce qu'elles ont été perçues comme le synonyme de la suppression partielle de l'accent circonflexe. Ce dernier a cristallisé une grande partie des inquiétudes et critiques. C'est en premier lieu cette suppression qui est concernée lorsque des informations fausses ou semi-correctes sont délivrées dans les articles de presse. À côté de ces erreurs qui peuvent contribuer à renforcer des idées erronées sur la réforme, nous noterons qu'une présentation intégrale de celle-ci n'a jamais lieu. L'information mise à la disposition des lecteurs ne concerne ainsi pas tellement et directement le contenu de la réforme mais davantage le débat qui a lieu autour d'elle. Au lieu de donner aux lecteurs la possibilité de se former leur propre opinion sur les rectifications, ce sont plutôt des avis - pour la plupart négatifs - tous faits d'institutions, de groupements et de personnes particulières - souvent des écrivains, à qui seuls on accorde le droit de juger de la < langue > - auxquels ces premiers se voient confrontés. Dans un même temps, les discours qui font preuve d'une attitude positive envers la réforme sont rares. En raison du nombre modéré d'articles publiés en 2009, la discussion du sujet se limite plus ou moins aux points mentionnés jusque-là. En ce qui concerne les années 1990 et 2016, deux autres thèmes sont mentionnés régulièrement. D'un côté, le souci que les rectifications et leur coexistence avec l'orthographe dite traditionnelle causent ou renforcent les inégalités sociales, les élites optant pour l'ancienne orthographe. Néanmoins, comme le montre

15. Les résultats de Vicari (2016) tendent à montrer une certaine symétrie entre les lignes éditoriales des deux journaux et leurs lecteurs. 
Vicari (2016: 115), le discours puriste ne se limite nullement aux articles de presse donnant la parole aux élites puisqu'il se retrouve dans les commentaires des quotidiens en ligne (Le Figaro), ce qui rend bien compte de son étendue au sein de la population. De l'autre côté, c'est la récurrence de la confusion entre langue et orthographe qui est à souligner. Le changement orthographique est ainsi perçu comme un mouvement naturel et libre et qui, s'il est imposé intentionnellement, contribue à une dégradation générale de la langue française.

À côté de ces points communs, il y a aussi quelques différences dans la discussion entre les trois moments. En 1990, quand la réforme vient d'être publiée, son caractère de décret venant du gouvernement cause une grande polémique. Le droit du gouvernement et des linguistes de légiférer sur la langue est largement remis en question. L'usage est souvent évoqué en tant que seul acteur valable. Cette idée réapparaît en 2016, mais à peu de reprises. Cette fois-ci, c'est surtout l'idée de la perte de l'histoire des mots et par conséquent la perte de l'histoire de la nation qui domine l'argumentation. En 2009, ce n'est pas tellement le sujet concret des rectifications qui est discuté mais celui d'une réforme orthographique en général.

Malgré des différences générales entre Le Figaro et Le Monde, on dénote une certaine constance des discours et des arguments présentés entre 1990 et 2016. Car sur cette même période, les rectifications de 1990 ont partiellement et par petites étapes gagné en importance ${ }^{16}$, le discours de cette presse écrite peut paraître en décalage avec la réalité des évènements. Par le prisme de la presse, cette hantise de réformer l'orthographe historiquement ancienne a pris l'ascendant sur une présentation complète, claire et plus objective des rectifications : le faible recours dans ces journaux aux linguistes et autres acteurs de la réforme dans sa justification, la priorité donnée à ses détracteurs, l'instabilité et le flou du positionnement de l'Académie française et du Ministère de l'éducation nationale semblent avoir alimenté, en plus d'en rendre compte, ce débat passionné sur cette réforme qui n'est toujours pas clos.

16. Voir leur intégration dans les dictionnaires ou dans les programmes scolaires, mentionnée plus haut. 


\section{Références}

Académie française (s.d.), Le français aujourd'hui. Du françois au français, disponible sur http://www.academie-francaise.fr/la-langue-francaise/le-francais-aujourdhui. [Page consultée le 10 mars 2018.]

Arrivé, Michel (1993), Réformer l’orthographe?, Paris, Presses universitaires de France.

Biedermann-Pasques, Liselotte (2006), « Un bilan des rectifications d'après une enquête en France et dans la francophonie (2002-2004) », dans Liselotte Biedermann-Pasques et Fabrice Jejcic (dir.), Les rectifications orthographiques de 1990. Analyses des pratiques réelles. Belgique, France, Québec, Suisse, 2002-2004, Orléans, Presses universitaires d’Orléans, p. 11-51.

Catach, Nina (1991), L'orthographe en débat, Paris, Nathan.

CIIP (Conférence intercantonale de l'instruction publique) (1996), Circulaire concernant la brochure "Les rectifications de l'orthographe du français », disponible sur http://www.orthographe-recommandee.info/enseignement/circulaire_suisse.pdf. [Page consultée le 9 juin 2018.]

Contant, Chantal (2009), Grand vadémécum de l'orthographe moderne recommandée. Cinq millepattes sur un nénufar, Montréal, Champlain.

Contant, Chantal et Romain Muller (2005), Connaitre et maitriser la nouvelle orthographe. Guide pratique et exercices, Montréal, Champlain.

CSLF (Conseil supérieur de la langue française du Québec) (2005) (dir.), Mémoire sur l'orthographe présenté par le Conseil supérieur de la langue française, disponible sur http://www.cslf.gouv.qc. ca/publications/pubf214/f214.pdf. [Page consultée le 9 juin 2018.]

De Closets, François (2009), Zéro faute. L'orthographe, une passion française, Paris, Mille et une nuits.

Feschet, Valérie (1998), « Les délices de l'orthographe ou la passion des Français pour « la dictée de Pivot », dans Christian Bromberger (dir.), Passions ordinaires. Du match de football au concours de la dictée, Paris, Bayard, 189-217.

Goosse, André (1991), La «nouvelle » orthographe, Paris/Louvain, Duculot.

Groupe RO (2012), «Les francophones et les rectifications orthographiques de 1990. État des connaissances et des usages en 2010 », Glottopol, no. 19, p. 130-148, disponible sur http://glottopol.univrouen.fr/numero_19.html. [Page consultée le 10 mars 2018.]

Honvault-Ducrocq, Renée (dir.) (2006), L'orthographe en questions, Mont-Saint-Aignan, Publications des Universités de Rouen et du Havre.

Keller, Monika (1991), Ein Jahrhundert Reformen der französischen Orthographie. Geschichte eines Scheiterns (1886 - 1991), Tübingen, Stauffenburg. 
Legros, Georges et Marie-Louise Moreau (2012), Orthographe: Qui a peur de la réforme?, disponible sur http://www.languefrancaise.cfwb.be/index.php?elD=tx_nawsecuredl\&u=0\&g=0\&hash=67d754f31f1924c7023e1262249b004e1c8ab83a\&file=fileadmin/sites/sgll/upload/lf_super_ editor/Docs/Ecrire_le_francais/Guide_ortho_HD.pdf. [Page consultée le 9 juin 2018.]

Masson, Michel (1991), L'orthographe. Guide pratique de la réforme, Paris, Seuil.

MELS (Ministère de l'Éducation, du Loisir et du Sport) (2009), Info/Sanction concernant les rectifications orthographiques et l'évaluation des apprentissages aux fins de la sanction des études secondaires, disponible sur http://www.nouvelleorthographe.info/mels_correction.pdf. [Page consultée le 9 juin 2018.]

Ministère de l'Éducation nationale (2008), Bulletin officiel du Ministère de l'Éducation nationale (spécial no. 6), « Programmes du collège. Programmes de l'enseignement de français ».

Ministère de l'Éducation nationale (2015), Bulletin officiel du Ministère de l'Éducation nationale (spécial no. 11), «Annexe 1 Programme d'enseignement du cycle des apprentissages fondamentaux (cycle 2) », disponible sur http://www.education.gouv.fr/pid285/bulletin_officiel.html?pid_ bo=33400. [Page consultée le 9 juin 2018.]

Ministère de l'Éducation nationale et Ministère de l'Enseignement supérieur et de la Recherche (2008), Bulletin officiel du Ministère de l'Éducation nationale et du Ministère de l'Enseignement supérieur et de la Recherche (hors-série no. 3), « Horaires et programmes d'enseignement de l'école primaire ».

Muller, Romain (2006), « Nouvelle orthographe et correcteurs informatiques avancés », dans Renée Honvault-Ducrocq (dir.), L'orthographe en questions, Mont-Saint-Aignan, Publications des Universités de Rouen et du Havre, 247-254.

RENOUVO (Réseau pour la nouvelle orthographe du français) (2005), Le millepatte sur un nénufar. Vadémécum de l'orthographe recommandée, Paris, De Champlain.

RENOUVO (dir.) (2006), Le point sur les Rectifications de l'orthographe en 2005, disponible sur www. renouvo.org/bilan2005.pdf. [Page consultée le 9 juin 2018.]

Vicari, Stefano (2016), "La réforme de l'orthographe dans les forums de discussion des articles du Monde, du Figaro et de Libération en ligne : que nous disent les non-linguistes ? ", Circula, no. 4, p. 106-125, disponible sur http://circula.recherche.usherbrooke.ca/wp-content/uploads/2017/03/2016_04_Vicari.pdf. [Page consultée le 10 mars 2018.]

Walter, Henriette (1994), L'aventure des langues en Occident, Paris, Robert Laffont. 